\title{
ST Elevation Myocardial Infarction by ECG
}

\section{Finding}

National Cancer Institute

\section{Source}

National Cancer Institute. ST Elevation Myocardial Infarction by ECG Finding. NCI

Thesaurus. Code 101888.

A myocardial infarction that produces elevation in the ST segments of the ECG. 